\title{
Dynamic Response of a Thick Piezoelectric Circular Cylindrical Panel: An Exact Solution
}

\author{
Atta Oveisi, Mohammad Gudarzi, and Seyyed Mohammad Hasheminejad \\ Department of Mechanical Engineering, Iran University of Science and Technology, Narmak, Tehran 1684613114, Iran \\ Correspondence should be addressed to Mohammad Gudarzi; gudarzi@iust.ac.ir
}

Received 26 September 2012; Accepted 19 November 2012; Published 27 May 2014

Academic Editor: Hamid Ahmadian

Copyright (C) 2014 Atta Oveisi et al. This is an open access article distributed under the Creative Commons Attribution License, which permits unrestricted use, distribution, and reproduction in any medium, provided the original work is properly cited.

\begin{abstract}
One of the interesting fields that attracted many researchers in recent years is the smart structures. The piezomaterials, because of their ability in converting both mechanical stress and electricity to each other, are very applicable in this field. However, most of the works available used various inexact two-dimensional theories with certain types of simplification, which are inaccurate in some applications such as thick shells while, in some applications due to request of large displacement/stress, thick piezoelectric panel is needed and two-dimensional theories have not enough accuracy. This study investigates the dynamic steady state response and natural frequency of a piezoelectric circular cylindrical panel using exact three-dimensional solutions based on this decomposition technique. In addition, the formulation is written for both simply supported and clamped boundary conditions. Then the natural frequencies, mode shapes, and dynamic steady state response of the piezoelectric circular cylindrical panel in frequency domain are validated with commercial finite element software (ABAQUS) to show the validity of the mathematical formulation and the results will be compared, finally.
\end{abstract}

\section{Introduction}

Piezoelectric materials have been extensively used as transducers and sensors due to their intrinsic direct and converse piezoelectric effects that take place between electric field and mechanical deformation. An important geometry in applied engineering problems is circular cylindrical panel because of its widespread application in actual structures such as aircraft wings, submarines, missiles, vessels, and high pressure cylindrical containers. The application of piezomaterial structures in this field is mainly concentrated on vibration suppression and acoustic noise reduction. Because of practical applications, piezoelectric circular cylindrical shells have attracted a considerable amount of research interests. Haskins and Walsh analyzed the free vibration of piezoelectric cylindrical shells with radially polarized transverse isotropy [1]; Martin investigated the vibration of longitudinally polarized piezoelectric cylindrical tubes and pointed out the limitations of the assumption [2]. Drumheller and Kalnins presented a coupled theory for the vibration of piezoceramic shells of revolution and analyzed the free axisymmetrical vibration of a circular cylindrical shell [3].
Burt simplified the circular cylinder to a two-dimensional model and then investigated the voltage response of radially polarized ceramic [4]. Tzou and Zhong gave a linear theory of piezoelectric shell vibration, which can be simplified to account for spheres [5]. Ebenezer and Abraham presented an Eigen function approach to determine the response of radially polarized piezoelectric cylindrical shells of finite length subjected to electrical excitation [6]. Many other researches by the methods of three-dimensional theory concentrated on the axisymmetrical and radial vibrations of cylinders, such as Stephenson [7, 8] and Adelman et al. [9, 10]. Paul derived the frequency equation of a piezoelectric cylindrical shell without presenting numerical results [11]. Paul and Venkatesan employed the same method to obtain the natural frequencies of infinite piezoelectric cylindrical shells [12]. However, some frequencies were missed in their calculation. Recently, Ding et al. exactly investigated the free vibration of hollow piezoelectric cylindrical shells on the basis of a decomposition formula for displacements, exactly [13]. Yang et al. considered the theory of the basic vibration characteristics of a circular cylindrical shell piezoelectric transducer [14]. They solved the vibration problem numerically for electrically 


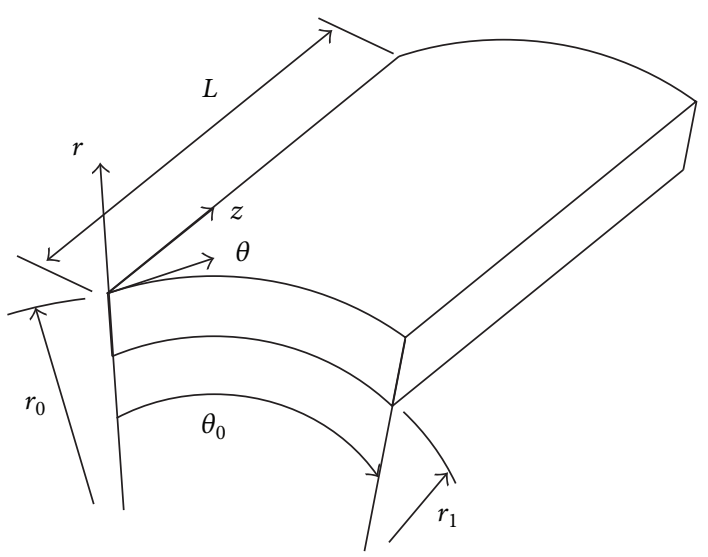

FIgURE 1: Cylindrical panel and its geometry.

forced case. Li et al. considered the spillover and harmonic effect in real active vibration control and they presented a novel composite controller based on disturbance observer (DOB) for the all-clamped panel [15]. Kumar and Singh aimed to examine through experiments vibration control of curved panel treated with optimally placed active or passive constrained layer damping patches and they found the optimum location for the application of ACLD/PCLD patches [16].

The main subject of this study is to investigate the free and forced vibration of transversely isotropic piezoelectric cylindrical panels. Based on the general solution for coupled equations for piezoelectric media presented in Ding et al. [17], three-dimensional exact solutions are obtained through the variable separation method. A numerical example is finally presented.

\section{Problem Formulation}

For dynamic modelling of piezoelectric layers, two displacement functions $\Psi$ and $F$ are considered [17]. Figure 1 shows the panel geometry.

2.1. Basic Equations. In circular cylindrical coordinates $(r, \theta, z)$, if the media is axially polarized the general solution can be written as

$$
\begin{gathered}
u_{r}^{P i}=\frac{1}{r} \frac{\partial \psi}{\partial \theta}-\frac{\partial}{\partial r} \mathscr{A}_{1} F, \\
u_{\theta}^{P i}=-\frac{\partial \Psi}{\partial r}-\frac{1}{r} \frac{\partial}{\partial \theta} \mathscr{A}_{1} F, \\
w^{P i}=\mathscr{A}_{2} F, \quad \phi=\mathscr{A}_{3} F,
\end{gathered}
$$

where $u_{r}^{P i}, u_{\theta}^{P i}$, and $w^{P i}$ are three displacement components, $\phi$ is the electric potential, and the differential operators $\mathscr{A}_{1}$, $\mathscr{A}_{2}$, and $\mathscr{A}_{3}$ are

$$
\begin{aligned}
\mathscr{A}_{1}= & {\left[\left(c_{13}^{P i}+c_{44}^{P i}\right) \varepsilon_{33}+\left(e_{15}+e_{31}\right) e_{33}\right] \frac{\partial^{3}}{\partial z^{3}} } \\
& +\left[\left(c_{13}^{P i}+c_{44}^{P i}\right) \varepsilon_{11}+\left(e_{15}+e_{31}\right) e_{15}\right] \Lambda \frac{\partial}{\partial z},
\end{aligned}
$$

$$
\begin{aligned}
\mathscr{A}_{2}= & c_{44}^{P i} \varepsilon_{33} \frac{\partial^{4}}{\partial z^{4}} \\
& +\left\{\left[c_{11}^{P i} \varepsilon_{33}+c_{44}^{P i} \varepsilon_{11}+\left(e_{15}+e_{31}\right)^{2} \Lambda-\rho \varepsilon_{33} \frac{\partial^{2}}{\partial t^{2}}\right]\right\} \frac{\partial^{2}}{\partial z^{2}} \\
& +c_{11}^{P i} \varepsilon_{11} \Lambda \Lambda-\rho \varepsilon_{11} \Lambda \frac{\partial^{2}}{\partial t^{2}}, \\
\mathscr{A}_{3}= & c_{44}^{P i} e_{33} \frac{\partial^{4}}{\partial z^{4}} \\
& +\left\{\left[c_{11}^{P i} e_{33}+c_{44}^{P i} e_{15}-\left(c_{13}^{P i}+c_{44}^{P i}\right)\left(e_{15}+e_{31}\right)\right] \Lambda\right. \\
& \left.-\rho e_{33} \frac{\partial^{2}}{\partial t^{2}}\right\} \frac{\partial^{2}}{\partial z^{2}} \\
& +c_{11}^{P i} e_{15} \Lambda \Lambda-\rho e_{15} \Lambda \frac{\partial^{2}}{\partial t^{2}},
\end{aligned}
$$

where $\Lambda=\partial^{2} / \partial r^{2}+(1 / r) \partial / \partial r+\left(1 / r^{2}\right) \partial^{2} / \partial \theta^{2}$ is the twodimensional Laplacian. The displacement functions $\Psi$ and $F$ must satisfy the following two equations:

$$
\begin{aligned}
\left(c_{66}^{\rho} \Lambda\right. & \left.+c_{44}^{P i} \frac{\partial^{2}}{\partial z^{2}}-\rho^{P i} \frac{\partial^{2}}{\partial t^{2}}\right) \psi=0, \quad L_{0} F=0, \\
L_{0}= & a_{4} \Lambda \Lambda \Lambda+\left(a_{3} \frac{\partial^{2}}{\partial z^{2}}+a_{6} \frac{\partial^{2}}{\partial t^{2}}\right) \Lambda \Lambda \\
& +\left(a_{2} \frac{\partial^{4}}{\partial z^{4}}+a_{5} \frac{\partial^{4}}{\partial t^{4}}+a_{7} \frac{\partial^{4}}{\partial z^{2} \partial t^{2}}\right) \Lambda+a_{1} \frac{\partial^{6}}{\partial z^{6}} \\
& +a_{8} \frac{\partial^{6}}{\partial z^{4} \partial t^{2}}+a_{9} \frac{\partial^{6}}{\partial z^{2} \partial t^{4}} .
\end{aligned}
$$


Here $a_{n}(n=1,2, \ldots, 9)$ can be expressed in terms of elastic constants $c_{i j}^{P i}$, dielectric constants $\varepsilon_{i j}$, and piezoelectric coefficients $e_{i j}$ as follows:

$$
\begin{gathered}
a_{1}=c_{44}^{P i}\left(e_{33}^{2}+c_{33}^{P i} \varepsilon_{33}\right), \\
a_{4}=c_{11}^{P i}\left(e_{15}^{2}+c_{44}^{P i} \varepsilon_{11}\right), \quad a_{5}=\rho^{2} \varepsilon_{11}, \\
a_{2}=c_{33}^{P i}\left[c_{44}^{P i} \varepsilon_{11}+\left(e_{15}+e_{31}\right)^{2}\right] \\
+\varepsilon_{33}\left[c_{11}^{P i} c_{33}^{P i}+c_{44}^{2}-\left(c_{11}^{P i}+c_{44}^{P i}\right)^{2}\right] \\
+e_{33}\left[2 c_{44}^{P i} e_{15}+c_{11}^{P i} e_{33}-2\left(c_{13}^{P i}+c_{44}^{P i}\right)\left(e_{15}+e_{31}\right)\right], \\
a_{3}=c_{44}^{P i}\left[c_{11}^{P i} \varepsilon_{33}+\left(e_{15}+e_{31}\right)^{2}\right] \\
+\varepsilon_{11}\left[c_{11}^{P i} c_{33}+c_{44}^{2}-\left(c_{13}^{P i}+c_{44}^{P i}\right)^{2}\right] \\
+e_{15}\left[2 c_{11}^{P i} e_{33}+c_{44}^{P i} e_{15}-2\left(c_{13}^{P i}+c_{44}^{P i}\right)\left(e_{15}+e_{31}\right)\right], \\
a_{6}=-\rho\left[e_{15}^{2}+\left(c_{11}^{P i}+c_{44}^{P i}\right) \varepsilon_{11}\right], \\
a_{8}=-\rho\left[e_{33}^{2}+\left(c_{44}^{P i}+c_{33}^{P i}\right) \varepsilon_{33}\right], \quad a_{9}=\rho^{2} \varepsilon_{33}, \\
a_{7}=-\rho\left[2 e_{15} e_{33}+\left(c_{44}^{P i}+c_{33}^{P i}\right) \varepsilon_{11}+\left(c_{11}^{P I}+c_{44}^{P i}\right) \varepsilon_{33}\right. \\
\left.+\left(e_{15}+e_{31}\right)^{2}\right] .
\end{gathered}
$$

The circular cylindrical coordinates as well as a circular cylindrical panel with outer radius $b^{P i}$, inner radius $a^{P i}$, circular center angle $\alpha^{P i}$, and length $L^{P i}$ are shown in Figure 1. If the panel is vibrating with a resonant frequency $\omega$, the displacement functions can be assumed as

$$
\begin{gathered}
F=\frac{r_{0}^{5}}{c_{11} \varepsilon_{33}} P\left(\xi^{P}\right) \Theta(\mu \theta) Z\left(\beta \zeta^{P}\right) e^{i \omega t}, \\
\psi=r_{0}^{2} P_{4}\left(\xi^{P}\right) \Theta^{\prime}(\mu \theta) Z^{\prime}\left(\beta \zeta^{P}\right) e^{i \omega t},
\end{gathered}
$$

where $\xi^{P i}=r^{P i} / R^{P i}, \zeta^{P i}=z^{P i} / L^{P i}$ are the dimensionless coordinates in $r$ and $z$ directions and $\Theta^{\prime}\left(\mu \theta^{P i}\right)$ and $Z^{\prime}\left(\beta \zeta^{P i}\right)$ denote the derivation of $\Theta\left(\mu \theta^{P i}\right)$ with respect to $\mu \theta^{P i}$ and the derivation of $Z\left(\beta \zeta^{P i}\right)$ with respect to $\beta \zeta^{P i}$, respectively. In addition,

$$
\begin{gathered}
\Theta(\mu \theta)=C_{1} \cos (\mu \theta)+C_{2} \sin (\mu \theta), \\
Z(\beta \zeta)=C_{3} \sin (\beta \zeta)+c_{4} \cos (\beta \zeta),
\end{gathered}
$$

where $C_{m}(m=1,2,3,4)$ are constants. Substitution of (6) into (3) yields

$$
\begin{gathered}
\left(\Delta+k_{4}^{2}\right) P_{4}(\xi)=0, \\
\left(\Delta+k_{1}^{2}\right)\left(\Delta+k_{2}^{2}\right)\left(\Delta+k_{3}^{2}\right) P_{4}(\xi)=0,
\end{gathered}
$$

where $\Delta=\partial^{2} / \partial\left(\xi^{P i}\right)^{2}+\left(1 / \xi^{P i}\right) \partial / \partial\left(\xi^{P i}\right)-\mu^{2} /\left(\xi^{P i}\right)^{2}$ and

$$
\begin{gathered}
k_{4}^{2}=\frac{\Omega^{2} c_{11}^{p i}}{c_{66}^{p i}}-\frac{\gamma^{2} c_{44}^{p i}}{c_{66}^{p i}}, \\
\left(\Omega^{P i}\right)^{2}=\frac{\rho^{P i} \omega^{2} r_{0}^{2}}{c_{11}}, \quad \gamma=\beta t_{1}, \quad t_{1}=\frac{r_{0}}{h_{0}}
\end{gathered}
$$

and $\left(k_{m}^{P i}\right)^{2},(m=1,2,3)$ (assuming $\left.\operatorname{Re}\left[k_{m}^{P i}\right] \geq 0\right)$ are the eigenvalues of the following equation:

$$
\begin{aligned}
\bar{a}_{4} k^{6}+ & \left(\bar{a}_{6}\left(\Omega^{P i}\right)^{2}+\bar{a}_{3} \gamma^{2}\right) k^{4} \\
& +\left(\bar{a}_{2} \gamma^{4}+\bar{a}_{7} \gamma^{2}\left(\Omega^{P i}\right)^{2}+\bar{a}_{5}\left(\Omega^{P i}\right)^{4}\right) k^{2} \\
& +\left(\bar{a}_{1} \gamma^{6}+\bar{a}_{8} \gamma^{4}\left(\Omega^{P i}\right)^{2}+\gamma^{2}\left(\Omega^{P i}\right)^{4}\right)=0
\end{aligned}
$$

in which

$$
\begin{gathered}
\bar{a}_{n}=\frac{a_{n}}{\left(\left(c_{11}^{P i}\right)^{2} \varepsilon_{33}\right)}, \quad(n=1,2,3,4), \\
\bar{a}_{n}=\frac{a_{n}}{\left(\rho c_{11}^{P i} \varepsilon_{33}\right)}, \quad(n=6,7,8), \\
\bar{a}_{5}=\frac{a_{5}}{\left(\left(\rho^{P i}\right)^{2} \varepsilon_{33}\right)} .
\end{gathered}
$$

The solution of (9) can be assumed as

$$
P(\xi)=P_{1}(\xi)+P_{2}(\xi)+P_{3}(\xi),
$$

where $P_{m}\left(\xi^{P i}\right)$ is obtained as [18].

Substituting (6) into (1) gives the mechanical displacements and electric potential as follows:

$$
u_{r}^{p i}=-r_{0}\left[\frac{\mu}{\xi} P_{4}(\xi)+\sum_{m=1}^{3} \alpha_{1 m} P_{m}^{\prime}(\xi)\right] \Theta(\mu \theta) Z^{\prime}(\beta \zeta) e^{i \omega t},
$$

$$
u_{\theta}^{p i}=-r_{0}\left[P_{4}^{\prime}(\xi)+\frac{\mu}{\xi} \sum_{m=1}^{3} \alpha_{1 m} P_{m}(\xi)\right] \Theta^{\prime}(\mu \theta) Z^{\prime}(\beta \zeta) e^{i \omega t},
$$

$$
w^{p i}=r_{0}\left[\sum_{m=1}^{3} \alpha_{2 m} P_{m}(\xi)\right] \Theta(\mu \theta) Z(\beta \zeta) e^{i \omega t},
$$

$$
\Phi=r_{0} \sqrt{\frac{c_{11}^{p i}}{\varepsilon_{33}}}\left[\sum_{m=1}^{3} \alpha_{3 m} P_{m}(\xi)\right] \Theta(\mu \theta) Z(\beta \zeta) e^{i \omega t},
$$


where

$$
\begin{aligned}
\alpha_{1 m}= & -\left(\left[\left(c_{13}^{P i}+c_{44}\right)\left(\varepsilon_{11} k_{m}^{2}+\varepsilon_{33} \gamma^{2}\right)\right.\right. \\
& \left.\left.+\left(e_{15}+e_{31}\right)\left(e_{15} k_{m}^{2}+e_{33} \gamma^{2}\right)\right] \gamma\right) \\
& \times\left(c_{11}^{P i} \varepsilon_{33}\right)^{-1}, \\
\alpha_{2 m}= & \left(\left[\left(c_{11}^{P i} k_{m}^{2}+c_{44}^{P i} \gamma^{2}-c_{11}^{P i} \Omega_{1}^{2}\right)\left(\varepsilon_{11} k_{m}^{2}+\varepsilon_{33} \gamma^{2}\right)\right.\right. \\
& \left.\left.+\left(e_{15}+e_{31}\right)^{2} k_{m}^{2} \gamma^{2}\right]\right) \\
& \times\left(c_{11}^{P i} \varepsilon_{33}\right)^{-1}, \\
\alpha_{3 m} & \left(\left[\left(c_{11}^{P i} k_{m}^{2}+c_{44}^{P i} \gamma^{2}-c_{11}^{P i} \Omega_{1}^{2}\right)\left(e_{15} k_{m}^{2}+e_{33} \gamma^{2}\right)\right.\right. \\
& \left.\left.\quad-\left(c_{13}^{P i}+c_{44}^{P i}\right)\left(e_{15}+e_{31}\right)^{2} k_{m}^{2} \gamma^{2}\right]\right) \\
& \times\left(c_{11}^{P i} \sqrt{c_{11}^{P i} \varepsilon_{33}}\right)^{-1}, \quad(m=1,2,3) .
\end{aligned}
$$

Utilizing the constitutive relations of piezoelectricity and (14)-(17), the stress components and electric displacement components can be derived as

$$
\begin{aligned}
\sigma_{r}^{p i}=\{ & \left(c_{12}^{p i}-c_{11}^{p i}\right)\left[\frac{\mu}{\xi} P_{4}^{\prime}(\xi)-\frac{\mu}{\xi^{2}} P_{4}(\xi)\right] \\
& +\left(c_{12}^{p i}-c_{11}^{p i}\right) \sum_{m=1}^{3} \alpha_{1 m} P_{m}^{\prime \prime}(\xi) \\
& +\sum_{m=1}^{3}\left(c_{12} \alpha_{1 m} k_{m}^{2}+c_{13}^{p i} \gamma \alpha_{2 m}+e_{31} \sqrt{\frac{c_{11}^{p i}}{\varepsilon_{33}} \gamma \alpha_{3 m}}\right) \\
& \left.\times P_{m}(\xi)\right\} \Theta(\mu \theta) Z^{\prime}(\beta \zeta) e^{i \omega t},
\end{aligned}
$$

$$
\begin{aligned}
& \sigma_{\theta}^{p i}=\left\{\left(c_{11}^{p i}-c_{12}^{p i}\right)\left[\frac{\mu}{\xi} P_{4}^{\prime}(\xi)-\frac{\mu}{\xi^{2}} P_{4}(\xi)\right]\right. \\
& +\left(c_{11}^{p i}-c_{12}^{p i}\right) \sum_{m=1}^{3} \alpha_{1 m} P_{m}^{\prime \prime}(\xi) \\
& +\sum_{m=1}^{3}\left(c_{11}^{p i} \alpha_{1 m} k_{m}^{2}+c_{13}^{p i} \gamma \alpha_{2 m}+e_{31} \sqrt{\frac{c_{11}^{p i}}{\varepsilon_{33}}} \gamma \alpha_{3 m}\right) \\
& \left.\times P_{m}(\xi)\right\} \Theta(\mu \theta) Z^{\prime}(\beta \zeta) e^{i \omega t},
\end{aligned}
$$

$$
\begin{aligned}
& \tau_{\theta z}^{p i}=\left\{c_{44}^{p i} \gamma P_{4}^{\prime}(\xi)\right. \\
& +\frac{\mu}{\xi} \sum_{m=1}^{3}\left[\left(c_{44}^{p i} \gamma \alpha_{1 m}+c_{44}^{p i} \alpha_{2 m}+e_{15} \sqrt{\frac{c_{11}^{p i}}{\varepsilon_{33}}} \alpha_{3 m}\right)\right. \\
& \left.\left.\times P_{m}(\xi)\right]\right\} \Theta^{\prime}(\mu \theta) Z(\beta \zeta) e^{i \omega t}
\end{aligned}
$$

$$
\begin{aligned}
\tau_{r z}^{p i}=\left\{c_{44}^{p i} \gamma \frac{\mu}{\xi} Q(\xi)\right. \\
+\left[\sum_{m=1}^{3}\left(c_{44}^{p i} \gamma \alpha_{1 m}+c_{44}^{p i} \alpha_{2 m}+e_{15} \sqrt{\frac{c_{11}^{p i}}{\varepsilon_{33}} \alpha_{3 m}}\right)\right. \\
\left.\left.\quad \times p_{M}^{\prime}(\xi)\right]\right\} \Theta(\mu \theta) Z(\beta \zeta) e^{i \omega t},
\end{aligned}
$$$$
\tau_{r \theta}^{p i}=c_{66}\left[-k_{4}^{2} P_{4}(\xi)-2 P_{4}^{\prime \prime}(\xi)\right.
$$$$
\left.+\frac{2 \mu}{\xi^{2}} \sum_{m=1}^{3} \alpha_{1 m} P_{m}(\xi)-\frac{2 \mu}{\xi} \sum_{m=1}^{3} \alpha_{1 m} p_{M}^{\prime}(\xi)\right]
$$$$
\times \Theta^{\prime}(\mu \theta) Z^{\prime}(\beta \zeta) e^{i \omega t}
$$$$
D_{r}^{p i}=\left\{e_{15} \gamma \frac{\mu}{\xi} P_{4}(\xi)\right.
$$$$
+\left[\sum_{m=1}^{3}\left(e_{15} \gamma \alpha_{1 m}+e_{15} \alpha_{2 m}+\varepsilon_{11} \sqrt{\frac{c_{11}^{p i}}{\varepsilon_{33}}} \alpha_{3 m}\right)\right.
$$$$
\left.\left.\times p_{M}^{\prime}(\xi)\right]\right\} \Theta(\mu \theta) Z(\beta \zeta) e^{i \omega t},
$$ 


$$
\begin{aligned}
D_{\theta}^{p i}= & \left\{e_{15} \gamma P_{4}^{\prime}(\xi)\right. \\
& +\frac{\mu}{\xi}\left[\sum_{m=1}^{3}\left(e_{15} \gamma \alpha_{1 m}+e_{15} \alpha_{2 m}+\varepsilon_{11} \sqrt{\frac{c_{11}^{p i}}{\varepsilon_{33}}} \alpha_{3 m}\right)\right. \\
\left.\left.\times P_{m}(\xi)\right]\right\} \Theta^{\prime}(\mu \theta) Z(\beta \zeta) e^{i \omega t}, & \\
D_{z}^{p i}= & {\left[\sum_{m=1}^{3}\left(e_{31} \alpha_{1 m} k_{m}^{2}+e_{33} \gamma \alpha_{2 m}-\sqrt{c_{11}^{p i} \varepsilon_{33}} \gamma \alpha_{3 m}\right) P_{m}(\xi)\right] } \\
& \times \Theta(\mu \theta) Z^{\prime}(\beta \zeta) e^{i \omega t} .
\end{aligned}
$$

2.2. Boundary Conditions. The piezoelectric panel has 8 boundary conditions consist of 6 mechanical and 2 electrical ones.

By considering generalized simply support boundary conditions at $\theta^{i}=0$ and $\theta^{i}=\alpha$ and $(i=P i)$ we will have

$$
w^{i}=u_{r}^{i}=0, \quad \sigma_{\theta}^{i}=0 \quad(i=P i) .
$$

Note that for piezoelectric layers the following condition is added

$$
\phi=0 .
$$

One can take

$C_{1}^{i}=0, \quad C_{2}^{i}=1, \quad \mu=\frac{(2 m+1) \pi}{2 \alpha}, \quad m=0,1,2, \ldots$

And by considering generalized simply support boundary conditions at $\zeta^{i}=0$ and $\zeta^{i}=1(i=P i)$ we will have

$$
u_{r}^{i}=u_{\theta}^{i}=0, \quad \sigma_{z}^{i}=0 \quad(i=P i) .
$$

And for piezoelectric layers the following condition is added

$$
D_{z}=0
$$

One can take

$$
C_{3}^{i}=0, \quad C_{4}^{i}=1, \quad \beta=n \pi, \quad n=0,1,2, \ldots .
$$

Without loss of generality, we suppose that external force acts on the outer surface of the actuator and inner surface of sensor has free boundary condition. So, we have

$$
\begin{gathered}
\sigma_{r}^{P i}=P, \quad \tau_{r \theta}^{P i}=\tau_{r z}^{P i}=0, \quad \phi=I \quad \text { at } r=r_{4}, \\
\sigma_{r}^{P i}=\tau_{r \theta}^{P i}=\tau_{r z}^{P i}=0, \quad \phi=0 \quad \text { at } r=r_{1} .
\end{gathered}
$$

For obtaining steady state frequency response of the cylindrical panel under a harmonic external excitation, we must solve the following matrix equation:

$$
[T]_{m \times n}\{X\}_{n \times 1}=\{F\}_{m \times 1},
$$

where $[T]_{m \times n}$ is the coefficient matrix. Consider

$$
\{X\}_{n \times 1}=\left[\begin{array}{llllllll}
A_{1} & B_{1} & A_{2} & B_{2} & A_{3} & B_{3} & A_{4} & B_{4}
\end{array}\right]
$$

and $A_{i}, B_{i}, i=1,2,3,4$ are the unknown constants that are in (19)-(27).

The vector $\{F\}_{m \times 1}$ denotes the force vector that acts on the structure. This force consists of the surface force that is considered as disturbance and has the breed of mechanical force such as wind effect. The effect of controller unit in the dynamic response of the piezo-panel is considered as an external electrical potential applied on the upper surface of the panel. These two external forces acted on the structure independently; however summation of their effects on the whole structure is the same as the case that both of them act on the structure simultaneously. So

$$
\begin{aligned}
& \{F\}_{n \times 2}=\left[\begin{array}{ll}
F_{1} & F_{2}
\end{array}\right], \\
& \left\{F_{1}\right\}_{n \times 1}=\left[\begin{array}{llllllll}
0 & 0 & 0 & 0 & 0 & 0 & I\left(r_{0}, \theta, z, \omega\right) & 0
\end{array}\right]^{T} \text {, }
\end{aligned}
$$

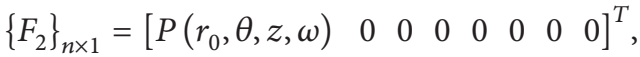

where $P\left(r_{0}, \theta, z, \omega\right)$ acting over the area $\left(L_{q} \leq x \leq L_{q}+a_{q}\right)$ on its top surface, while it is traction-free at the bottom surface. Thus

$$
\begin{aligned}
& P(r, \theta, z, \omega)=\sum_{n=-\infty}^{\infty} \sum_{m=0}^{\infty} p_{n m}(r, \omega) \operatorname{Sin}\left(\frac{m \pi z}{l}\right) e^{i(n \theta+\omega t)}, \\
& I(r, \theta, z, \omega)=\sum_{n=-\infty}^{\infty} \sum_{m=0}^{\infty} \iota_{n m}(r, \omega) \operatorname{Sin}\left(\frac{m \pi z}{l}\right) e^{i(n \theta+\omega t)},
\end{aligned}
$$

where

$$
\begin{aligned}
p_{n m}(r, \omega) & = \begin{cases}A_{n m}(\omega) J_{n}(K r), & K^{2}>0, \\
A_{n m}(\omega) r^{n}, & K^{2}=0, \\
A_{n m}(\omega) I_{n}(\bar{K} r), & K^{2}=-\bar{K}^{2}<0,\end{cases} \\
\iota_{n m}(r, \omega) & = \begin{cases}B_{n m}(\omega) J_{n}(K r), & K^{2}>0, \\
B_{n m}(\omega) r^{n}, & K^{2}=0, \\
B_{n m}(\omega) I_{n}(\bar{K} r), & K^{2}=-\bar{K}^{2}<0\end{cases}
\end{aligned}
$$

in which $K=\sqrt{k^{2}-(\pi m / L)^{2}}$ and $J_{n}$ and $I_{n}$ denote the standard and modified cylindrical Bessel functions of first kind, respectively, and $A_{n m}(\omega)$ and $B_{n m}(\omega)$ are the amplitude of the applied forces. Substituting (21), (25), and (26) into the mechanical condition (39) and substituting (27) or (19) into the electric condition (40) yields homogeneous equations with respect to coefficients $A_{m}$ and $B_{m},(m=1,2,3,4)$. After finding these unknown constants that are functions of 


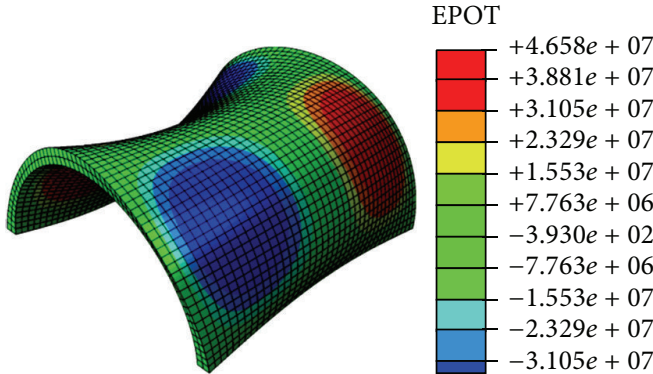

(a) First mode shape

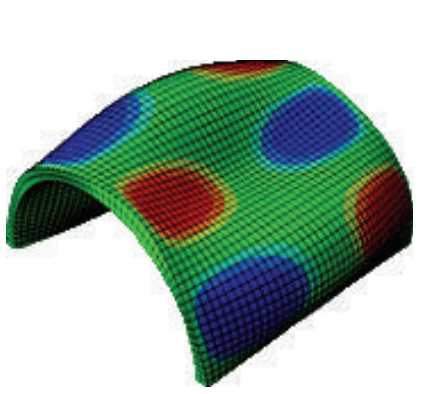

(c) Third mode shape

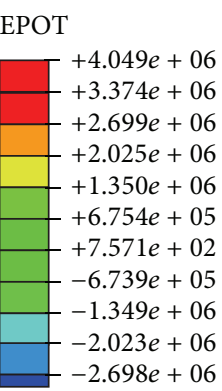

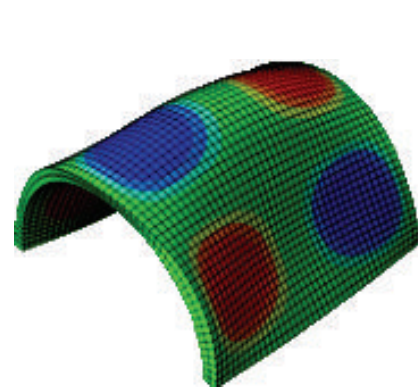

EPOT

(b) Second mode shape

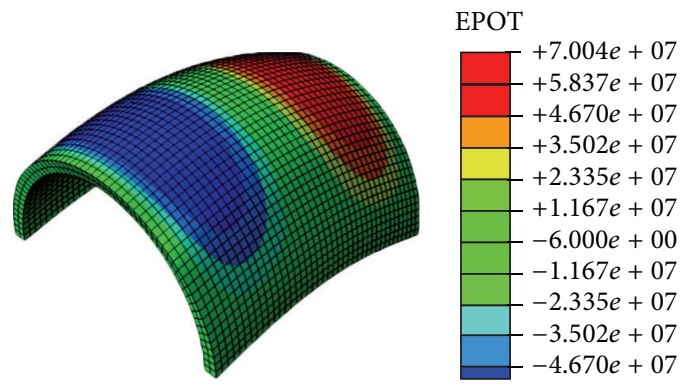

(d) Forth mode shape

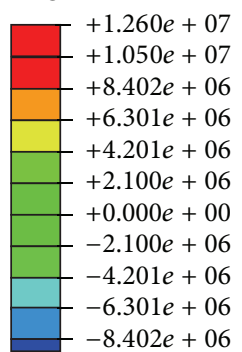

$-8.402 e+06$
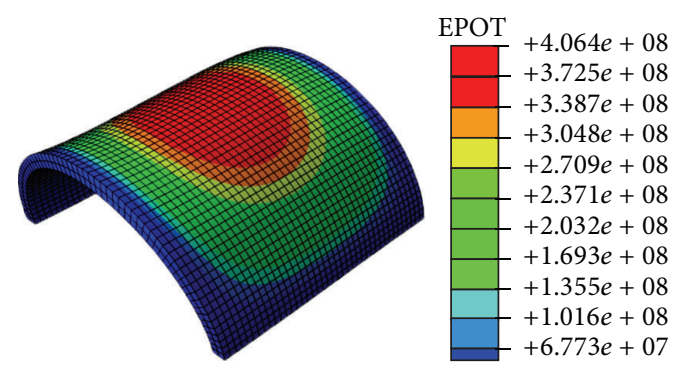

(e) Fifth mode shape

FIgURE 2: Mode shapes of the five first natural frequencies.

$m, n$ by replacing them in the displacement and stress and electric displacement of corresponding equations (19)-(27), all of the system variables will be determined easily. However for control purposes the voltage obtained from the piezolayer as a sensor is the measured output and it is calculated as

$$
q=\int_{\text {Area }} \bar{D} \cdot d A_{\text {Area }}
$$

where $\bar{D}=D_{r} \widehat{r}+D_{\theta} \widehat{\theta}+D_{z} \widehat{z}$ is the electric displacement vector in the principle cylindrical coordinates. Area in the integration stands for the place that the sensor layer is active and voltage (control output) is measured and $d A_{\text {Area }}=(d z \times$ $d \theta) \hat{r}$ which simplifies the above equation as

$$
q_{r}=\int_{\theta_{1}^{s}}^{\theta_{2}^{s}} \int_{z_{1}^{s}}^{z_{2}^{s}} D_{r} d z d \theta
$$

Moreover, by considering the piezoelectric sensor layer as an electric capacity $V=q / c_{P S}$, one can obtain

$$
V=\frac{1}{c_{P S}} \int_{\theta_{1}^{s}}^{\theta_{2}^{s}} \int_{z_{1}^{s}}^{z_{2}^{s}} D_{r} d z d \theta
$$

where $c_{P S}$ is the capacitance of the piezoelectric sensor.
TABLE 1: First three nondimensional natural frequencies.

\begin{tabular}{lllllll}
\hline$S$ & \multicolumn{3}{c}{$\mu=1.8$} & & $\mu=0.9$ & \\
\hline 0.1 & 0.9366 & 1.8562 & 2.3634 & 0.5178 & 1.4580 & 1.8160 \\
0.2 & 0.8266 & 1.7995 & 2.3514 & 0.5109 & 1.4144 & 1.8214 \\
0.3 & 0.7214 & 1.7650 & 2.3616 & 0.5043 & 1.3565 & 1.8434 \\
0.4 & 0.6271 & 1.7193 & 2.3889 & 0.4975 & 1.2960 & 1.8666 \\
0.5 & 0.5408 & 1.6549 & 2.4273 & 0.4906 & 1.2385 & 1.8741 \\
\hline
\end{tabular}

\section{Results and Discussion}

Table 1 shows the first three nondimensional natural frequencies of some panels by different geometries. Mode shapes of the five first natural frequencies are shown in Figure 2. The panel dynamic responses under the aforementioned inputs (dynamic excitation and electric excitation) are shown in Figure 3 and are compared by FEM results.

It is obvious that a good accommodation exist between analytical solution and FEM (ABAQUS) method. In addition, the dynamic response of the panel in $450 \mathrm{~Hz}$ is shown in 


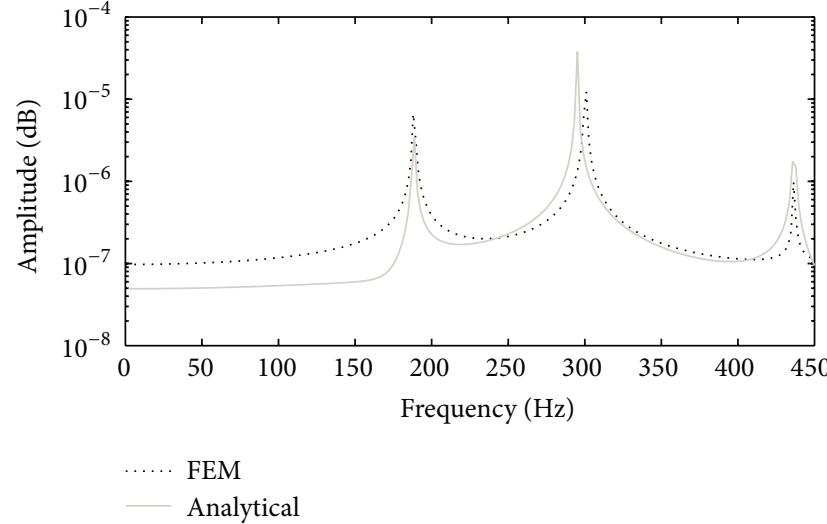

(a)

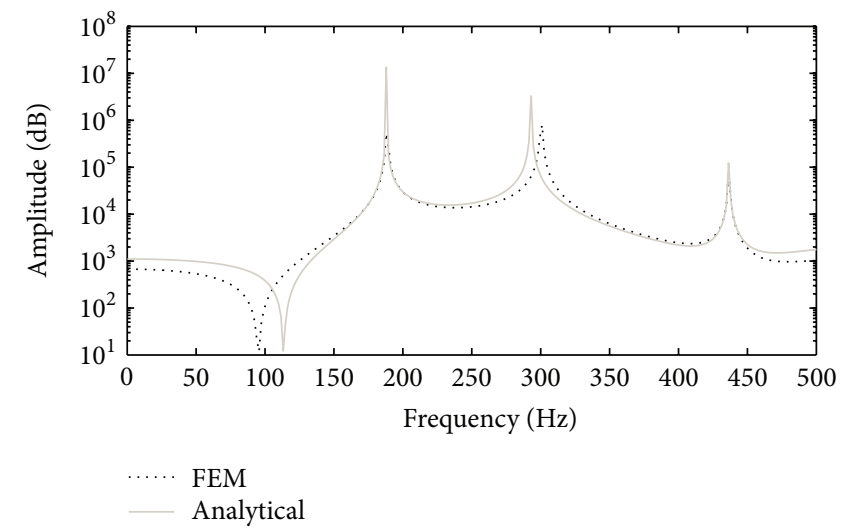

(b)

FIgure 3: Panel dynamic response. (a) Mechanical excitation; (b) electrical excitation.

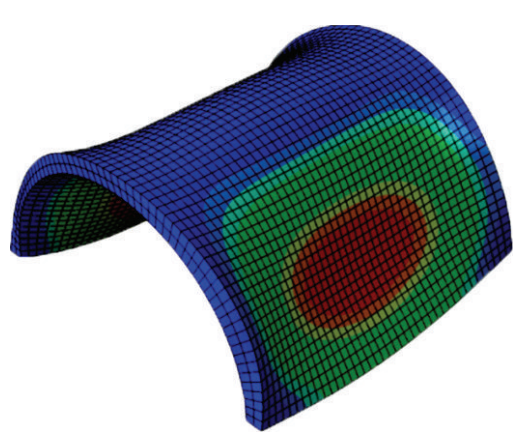

(a)

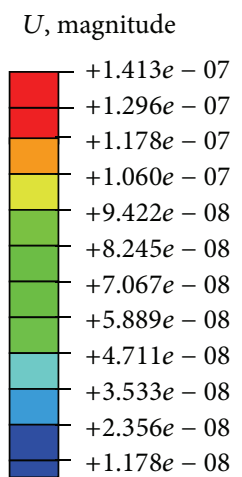

$+1.178 e-08$

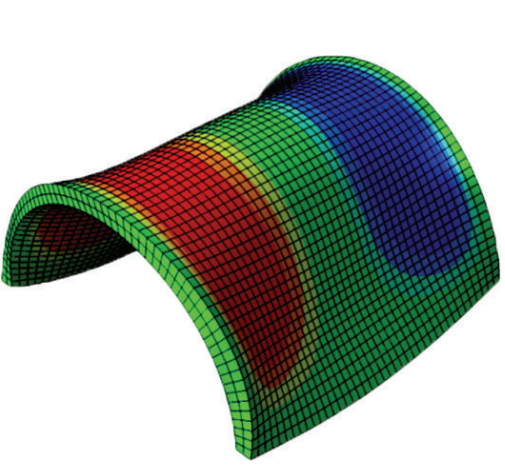

(b)

Figure 4: The dynamic response of the panel at $450 \mathrm{~Hz}$ due to (a) mechanical excitation; (b) electrical excitation.

Figure 4. It can be seen that the dominant mode shape in this frequency is the third mode shape.

\section{Conclusion}

Based on the general solution of the coupled equations for a piezoelectric media, the displacement functions are expanded in terms of trigonometric functions in $z$ and $\theta$ directions. Three-dimensional exact solutions for the free vibration of a piezoelectric circular cylindrical panel are then obtained under several boundary conditions. Also the forced vibration is solved. The natural frequencies are compared with previous works. The dynamic responses with mechanical and electrical excitation are validated with FEM and the mode shapes are shown.

\section{Conflict of Interests}

The authors declare that there is no conflict of interests regarding the publication of this paper.

\section{References}

[1] J. F. Haskins and J. L. Walsh, "Vibrations of ferroelectric cylindrical shells with transverse isotropy: I. Radially polarized case," The Journal of the Acoustical Society of America, vol. 29, no. 6, pp. 729-734, 1975.

[2] G. E. Martin, "Vibrations of longitudinally polarized ferroelectric cylindrical tubes," The Journal of the Acoustical Society of America, vol. 35, no. 4, pp. 510-520, 1963.

[3] D. S. Drumheller and A. Kalnins, "Dynamic shell theory for ferroelectric ceramics," The Journal of the Acoustical Society of America, vol. 47, no. 5, pp. 1343-1353, 1970.

[4] J. A. Burt, "The electroacoustic sensitivity of radially polarized ceramic cylinders as a function of frequency," The Journal of the Acoustical Society of America, vol. 64, no. 6, pp. 1640-1644, 1978.

[5] H. S. Tzou and J. P. Zhong, "A linear theory of piezoelastic shell vibrations," Journal of Sound and Vibration, vol. 175, no. 1, pp. 77-88, 1994.

[6] D. D. Ebenezer and P. Abraham, "Eigenfunction analysis of radially polarized piezoelectric cylindrical shells of finite length," The Journal of the Acoustical Society of America, vol. 102, no. 3, pp. 1549-1558, 1997. 
[7] C. V. Stephenson, "Radial vibrations in short, hollow cylinders of barium titanate," The Journal of the Acoustical Society of America, vol. 28, no. 1, pp. 51-56, 1956.

[8] C. V. Stephenson, "Higher modes of radial vibrations in short, hollow cylinders of barium titanate," The Journal of the Acoustical Society of America, vol. 28, no. 5, pp. 928-929, 1956.

[9] N. T. Adelman, Y. Stavsky, and E. Segal, "Axisymmetric vibrations of radially polarized piezoelectric ceramic cylinders," Journal of Sound and Vibration, vol. 38, no. 2, pp. 245-254, 1975.

[10] N. T. Adelman, Y. Stavsky, and E. Segal, "Radial vibrations of axially polarized piezoelectric ceramic cylinders," The Journal of the Acoustical Society of America, vol. 57, no. 2, pp. 356-360, 1975.

[11] H. S. Paul, "Vibrations of circular cylindrical shells of piezoelectric silver iodide crystals," The Journal of the Acoustical Society of America, vol. 40, no. 5, pp. 1077-1080, 1966.

[12] H. S. Paul and M. Venkatesan, "Vibrations of a hollow circular cylinder of piezoelectric ceramics," The Journal of the Acoustical Society of America, vol. 82, no. 3, pp. 952-956, 1987.

[13] H.-J. Ding, W.-Q. Chen, Y.-M. Guo, and Q.-D. Yang, "Free vibrations of piezoelectric cylindrical shells filled with compressible fluid," International Journal of Solids and Structures, vol. 34, no. 16, pp. 2025-2034, 1997.

[14] Z. Yang, J. Yang, Y. Hu, and Q.-M. Wang, "Vibration characteristics of a circular cylindrical panel piezoelectric transducer," IEEE Transactions on Ultrasonics, Ferroelectrics, and Frequency Control, vol. 55, no. 10, pp. 2327-2335, 2008.

[15] S. Li, J. Qiu, H. Ji, K. Zhu, and J. Li, "Piezoelectric vibration control for all-clamped panel using DOB-based optimal control," Mechatronics, vol. 21, no. 7, pp. 1213-1221, 2011.

[16] N. Kumar and S. P. Singh, "Vibration control of curved panel using smart damping," Mechanical Systems and Signal Processing, vol. 30, pp. 232-247, 2012.

[17] H. J. Ding, B. Chen, and J. Liang, "General solutions for coupled equations for piezoelectric media," International Journal of Solids and Structures, vol. 33, no. 16, pp. 2283-2298, 1996.

[18] H. J. Ding, R. Q. Xu, and W. Q. Chen, "Free vibration of transversely isotropic piezoelectric circular cylindrical panels," International Journal of Mechanical Sciences, vol. 44, no. 1, pp. 191-206, 2002. 

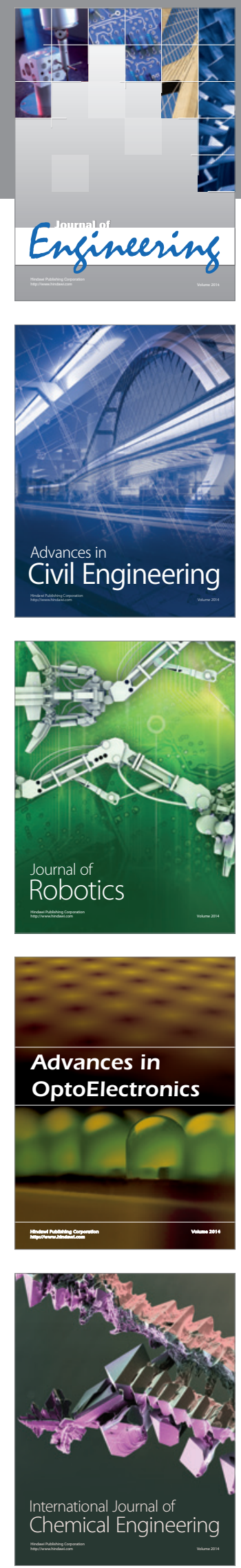

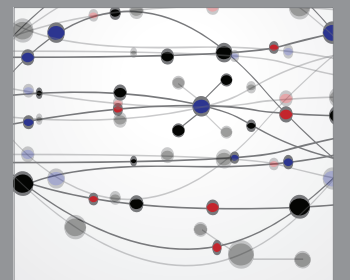

The Scientific World Journal
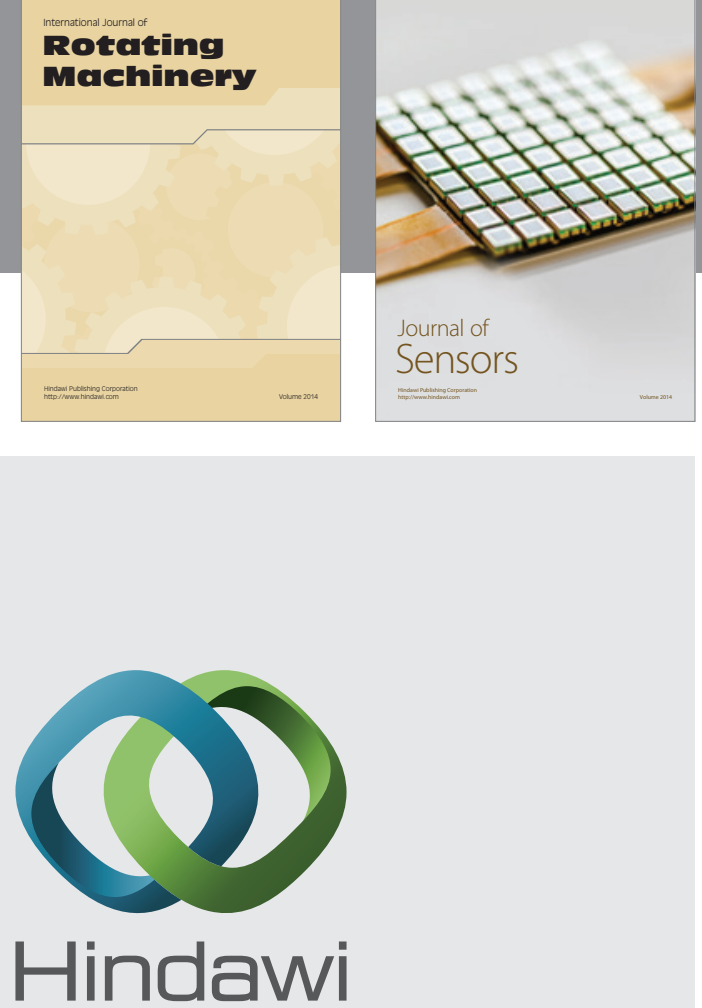

Submit your manuscripts at http://www.hindawi.com
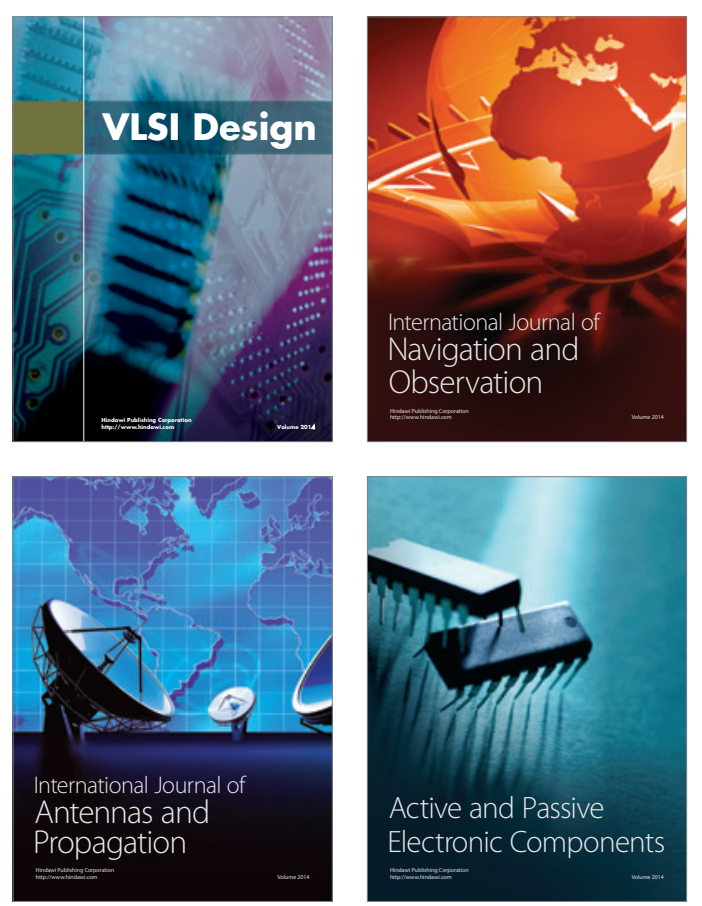
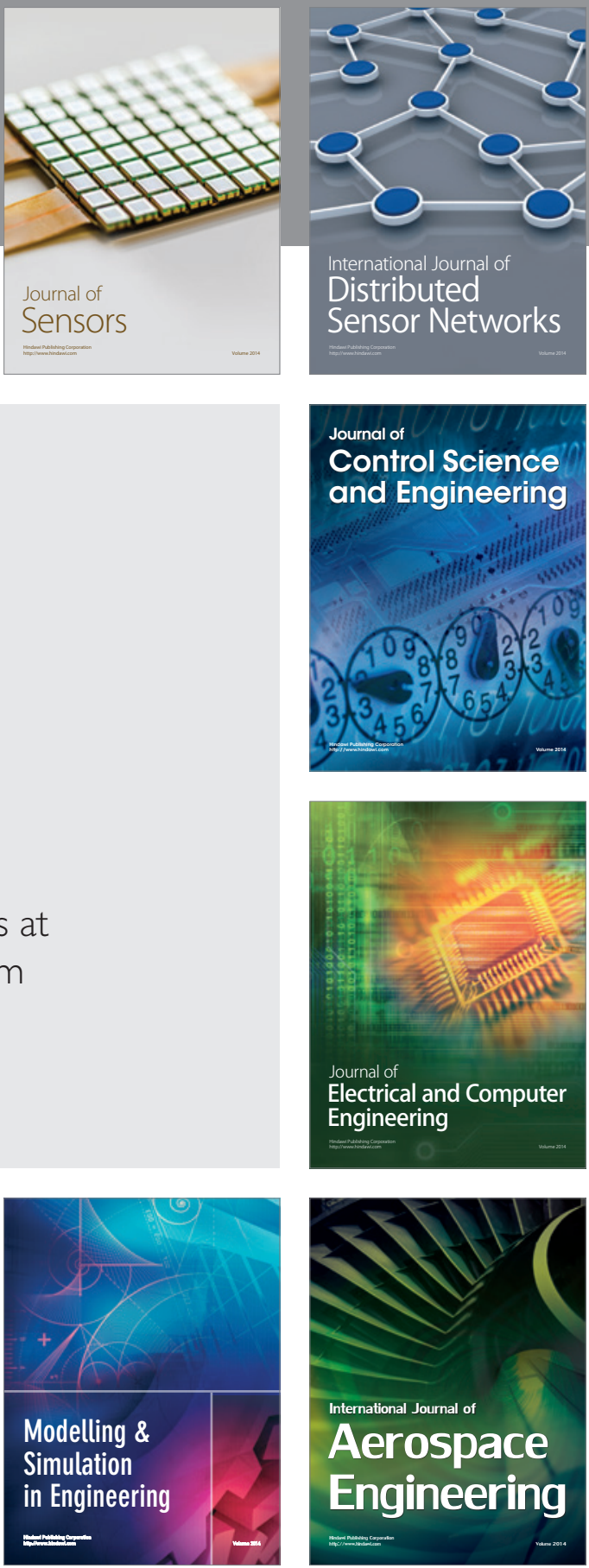

Journal of

Control Science

and Engineering
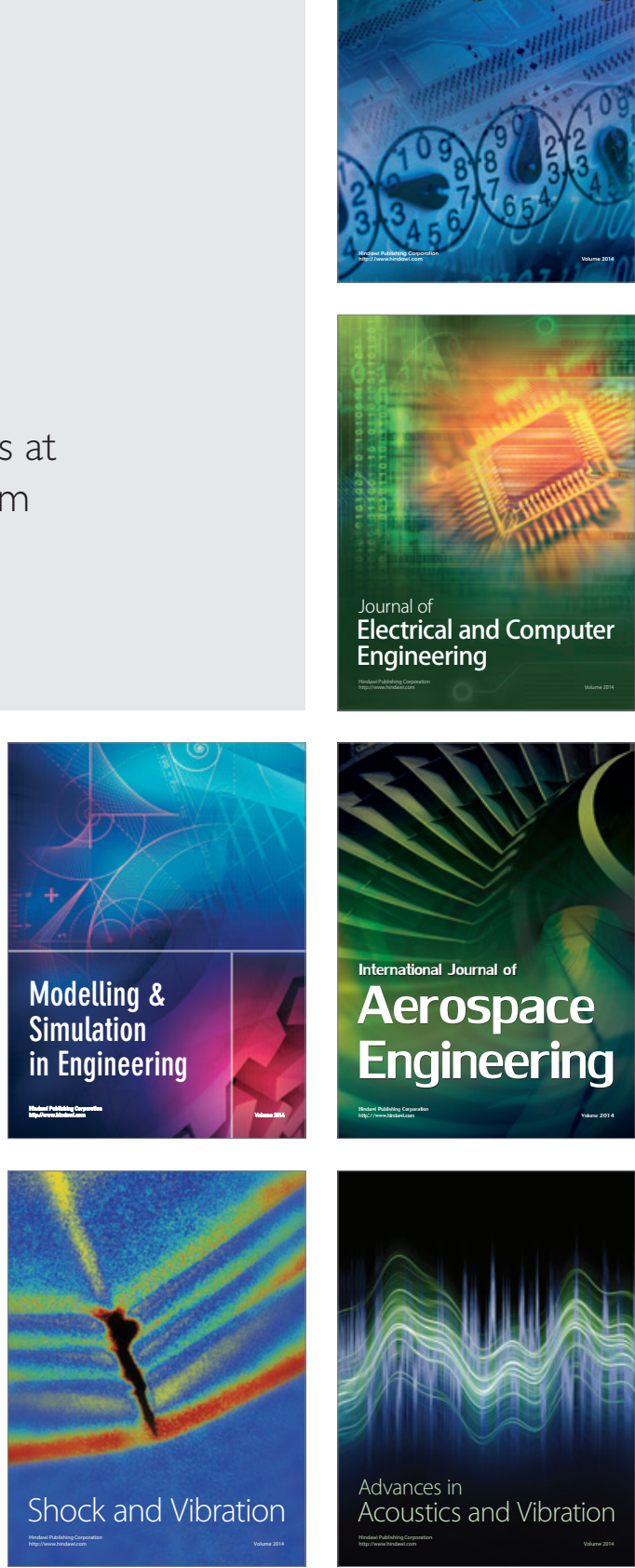